\title{
Prehospital Use of Ketamine: A Systematic Review
}

\author{
Leonard Ranasinghe*, Jessica Doctor MS and Radhika Gulhar MS \\ Professor of Medical Education and Emergency Medicine, California Northstate University, USA
}

*Corresponding author: Leonard Ranasinghe, Professor of Medical Education and Emergency Medicine, California Northstate University, USA.

To Cite This Article: Leonard Ranasinghe, Prehospital Use of Ketamine: A Systematic Review. Am J Biomed Sci \& Res. 2020 - 7(1). AJBSR. MS.ID.001112. DOI: 10.34297/AJBSR.2020.07.001112.

Received: 阱 December 17, 2019; Published: 眥 January 27, 2020

\section{Background}

Acute pain and agitation are common presentations that must be addressed in the prehospital setting by paramedics. Opioids are most frequently used to control acute pain but are highly addictive and patients can develop dependence even from short term use. For sedation, many different medications such as benzodiazepines and haloperidol are used, but their delayed onset of action is not desirable and side effects at effective doses (e.g. CNS depression) can be potentially harmful to the patients. In recent years, ketamine has been used as a safer alternative for prehospital analgesia and sedation in the military setting, but has not been studied sufficiently in the civilian setting to be a part of standard protocol nationwide [1]. Ketamine's primary action is to block glutamate from acting on the n-Methyl-d-Aspartate (NMDA) receptor resulting in a "dissociative anesthesia" state. It also works on other cellular processes such as hyperpolarization-activated cyclic nucleotide channels (HCN1), cholinergic, aminergic, and opioid systems making it useful for both analgesia and sedation [2].

\section{Method}

A review of recent literature from multiple electronic databases with the keywords "prehospital ketamine use" was conducted. The oldest article dates back to May 2013 while the most recent article was published March 2019.

\section{Results}

The articles reviewed either focused on the prehospital effectiveness of ketamine or compared ketamine to other widely used drugs. Ketamine has been shown to be effective when used alone or in conjunction with other drugs for a variety of injuries and comorbid conditions [1]. It can be used without intravenous access for rapid and safe sedation of violent and agitated patients with low respiratory or hemodynamic side effects $[1,3,4]$. Ketamine can be administered intramuscularly and works within three to four minute [4]. In a study done in 2018 comparing IV prehospital analgesics it was observed that ketamine provided a significant reduction in pain by $50 \%$ with a lack of adverse effects in comparison to fentanyl [5].

Another study done in 2016 measured that the median time to adequate sedation was 12 minutes faster with IM ketamine as compared to IM haloperidol for severe prehospital acute agitation. It was also noted that the only disadvantage of ketamine was higher intubation rates in comparison to haloperidol [6]. A third study in 2015 done in Iraq looked at the use of prehospital analgesia in rural trauma systems for serious injury patients. It was observed that ketamine had a significant better effect on improving systolic blood pressure as compared to pentazocine [7]. A study in 2018 looked at pain reduction using nitrous oxide with added intranasal ketamine versus intranasal steroids for hospital patients. It was reported the verbal numeric rating scale (VNRS) was significantly reduced after 30 minutes for the patients administered intranasal ketamine [8].

Despite its apparent effectiveness and safety, a large national survey of paramedics in the United States was conducted in 2018 that showed training related to use of ketamine was common, but few were authorized to use it by their agency's protocol and even fewer had experience administering it [9]. Australia and New Zealand have been studying and using ketamine on the field by paramedics for several years. They have found decreased rates in intubation and pain scores after ketamine was introduced into their protocol [10-12].

\section{Conclusion}

Ketamine administration is a more rapid and effective alternative to achieve analgesia and sedation in the prehospital setting with few clinical side effects. In comparison to other prehospital analgesia opioid like fentanyl and haloperidol it showed increased efficacy in reducing pain and improving blood pressure. It has yet to be introduced into prehospital protocol nationwide in the United States but has been part of protocol for years in Australia and New Zealand with overall improved outcomes in pain and intubation rates. 


\section{References}

1. John Zietlow, Kathy Berns, Donald Jenkins, Scott Zietlow (2019) Prehospital Use of Ketamine: Effectiveness in Critically Ill and Injured Patients. Military Medicine 184(1): 542-544.

2. Jamie Sleigh, Martyn Harvey, Logan Voss, Bill Denny (2014) Ketamine - More mechanisms of action than just NMDA blockade. Trends in Anaesthesia and Critical Care 4(2-3): 76-81.

3. Scheppke KA, Braghiroli J, Shalaby M, Chait R (2014) Prehospital use of i.m. ketamine for sedation of violent and agitated patients. West J Emerg Med 15(7): 736-741.

4. Scaggs TR, Glass DM, Hutchcraft MG, Weir WB (2016) Prehospital Ketamine is a Safe and Effective Treatment for Excited Delirium in a Community Hospital Based EMS System. Prehosp Disaster Med 31(5): 563-569.

5. Bronsky EStein, Koola C, Orlando A, Redmond D, D’Huyvetter Cm, et al. (2018) Intravenous Low-Dose Ketamine Provides Greater Pain Control Compared to Fentanyl in a Civilian Prehospital Trauma System: A Propensity Matched Analysis. Prehosp Emerg Care 23(1): 1-8.

6. Cole Jon B, Johanna C Moore, Paul C Nystrom, Benjamin S, et al. (2016) A Prospective Study of Ketamine versus Haloperidol for Severe Prehospital Agitation. Clinical Toxicology 54(7): 556-562.

7. Losvik Ole Kristian, Mudhafar Kareem Murad, Eystein Skjerve, Hans Husum (2015) Ketamine for Prehospital Trauma Analgesia in a Low-
Resource Rural Trauma System: a Retrospective Comparative Study of Ketamine and Opioid Analgesia in a Ten-Year Cohort in Iraq. Scandinavian Journal of Trauma, Resuscitation and Emergency Medicine 23(1).

8. Andolfatto Gary, Innes K, Dick W, Jenneson S, Willman E, et al. (2019) Prehospital Analgesia With Intranasal Ketamine (PAIN-K): A Randomized Double-Blind Trial in Adults. Annals of Emergency Medicine 74(2): 241250 .

9. Buckland DM, Crowe RP, Cash RE, Stephen Gondek, Patrick Maluso, et al. (2018) Ketamine in the Prehospital Environment: A National Survey of Paramedics in the United States. Prehospital and Disaster Medicine 33(1): 23-28.

10. Hollis, Gregory, Keene, Toby, Ardlie, et al. (2016) Prehospital ketamine use by paramedics in the Australian Capital Territory: A12 month retrospective analysis: Prehospital Ketamine use by Paramedics. Emergency Medicine Australasia.

11. Jennings PA, Cameron P, Bernard, Walker T, Jolley D S, et al. (2014) Long-term pain prevalence and health-related quality of life outcomes for patients enrolled in a ketamine versus morphine for prehospital traumatic pain randomised controlled trial. Emerg Med J 31: 840-843.

12. Shahtahmasebi, R Johnson R, Shahtahmasebi S (2019) Impact of a ketamine sedation protocol on intubation rates and undesirable outcomes in the transport of patients with acute behavioural disturbance. Emerg Med Australas. 